\title{
« A dream turned to ashes »? Les évolutions contradictoires du nationalisme gallois dans les années 1970
}

The Contradictions of Welsh Nationalism in the 1970s

\section{Stéphanie Bory}

\section{(2) OpenEdition}

\section{Journals}

Édition électronique

URL : http://journals.openedition.org/rfcb/1714

DOI : $10.4000 /$ rfcb. 1714

ISSN : 2429-4373

Éditeur

CRECIB - Centre de recherche et d'études en civilisation britannique

Référence électronique

Stéphanie Bory, " «A dream turned to ashes »? Les évolutions contradictoires du nationalisme gallois dans les années 1970 », Revue Française de Civilisation Britannique [En ligne], XXII- Hors série | 2017, mis en ligne le 30 décembre 2017, consulté le 20 avril 2019. URL : http://journals.openedition.org/ rfcb/1714 ; DOI : 10.4000/rfcb.1714

Ce document a été généré automatiquement le 20 avril 2019.

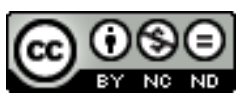

Revue française de civilisation britannique est mis à disposition selon les termes de la licence Creative Commons Attribution - Pas d'Utilisation Commerciale - Pas de Modification 4.0 International. 


\title{
«A dream turned to ashes »? Les évolutions contradictoires du nationalisme gallois dans les années $1970^{1}$
}

\author{
The Contradictions of Welsh Nationalism in the 1970s
}

\section{Stéphanie Bory}

1 C'est le 5 août 1925 à Pwllheli, dans le Caernarvonshire, au cours de l'eisteddfod², que le parti nationaliste gallois voit le jour, né de la fusion de deux groupements nationalistes existants. Six hommes, la plupart désabusés par la Première Guerre mondiale, parmi lesquels Saunders Lewis, universitaire, et Lewis Valentine, pasteur, décident de former Plaid Genedlaethol Cymru, le premier parti politique nationaliste indépendant de l'histoire du pays de Galles moderne. Ils souhaitent donner cohésion à un mouvement diffus et peu efficace afin de construire "un pays de Galles parlant gallois ${ }^{3}$ ", administré par des autorités locales, et définissent trois objectifs prioritaires : faire en sorte que le gallois soit reconnu seule langue officielle, qu'il soit l'unique langue utilisée dans la vie économique et par l'administration, ainsi que le seul vecteur de l'instruction à tous les niveaux. Plaid Cymru fonctionne dans un premier temps davantage comme un groupe de pression, mais l'arrivée de Gwynfor Evans à la tête du parti en 1945 lui permet d'acquérir une nouvelle dimension et il conçoit un véritable projet économique et politique.

2 Les années 1960 sont le théâtre d'une montée du nationalisme gallois qui remporte plusieurs victoires : la création, en 1964, du Welsh Office, et l'élection, le 14 juillet 1966, du premier député nationaliste, Evans, dans la circonscription de Carmarthen. C'est une date particulièrement symbolique puisqu'elle marque la prise de la Bastille en France en 1789, et le $80^{\mathrm{e}}$ anniversaire de l'élection de Thomas Ellis, l'un des fondateurs de Cymru Fydd ${ }^{4}$ à la fin du XIX ${ }^{\mathrm{e}}$ siècle et auteur d'un discours célèbre prononcé à Bala en 1890 dans lequel il réclame la création d'une assemblée législative pour le pays de Galles. La victoire d'Evans est saluée par le poète Gwenallt dans son sonnet "Sir Gaerfyrddin », publié dans Y Draig Goch en septembre 1967, comme « l'aube nationaliste ${ }^{5}$ » et le Daily Mail titre le 15 juillet « 1 
a.m.: The Welsh Unseat Labour ». Plaid Cymru semble enfin être devenu une véritable force politique. Au cours des trois jours qui suivent la victoire d'Evans, il accueille 700 nouveaux membres et double en une année, passant de 14000 à 27000 membres $^{6}$. Lors de son entrée à Westminster, Evans demande le droit de prêter serment en gallois, après l'avoir fait en anglais. Horace King, le Speaker de la Chambre des Communes, refuse mais seulement après avoir obtenu la promesse du Leader de reconsidérer le règlement. C'est chose faite en 1970 lorsque décision est prise d'autoriser les députés gallois à prêter serment en anglais et en gallois. De plus, le Parlement adopte en 1967 le Welsh Language Act, instaurant l'égalité de l'anglais et du gallois même si le gallois n'est pas reconnu langue officielle. Enfin, dès 1968, le Premier ministre travailliste Harold Wilson met en place une commission d'enquête sur la constitution ${ }^{7}$ chargée d'étudier les modalités d'une éventuelle dévolution, dirigée tout d'abord par Geoffrey Crowther, journaliste économique, puis, après son décès, par Lord Kilbrandon. La commission enquête de 1968 à 1973 avant de publier ses conclusions ${ }^{8}$.

3 Les années 1970 semblent alors débuter sous les meilleurs auspices : Plaid Cymru remporte des victoires tant électorales que politiques. Pourtant, le parti est divisé sur plusieurs sujets et souffre de dissensions internes, ce qui contribue à le fragiliser au pays de Galles. Le nationalisme gallois se retrouve donc face à un paradoxe : Plaid Cymru semble être plus influent et puissant au Parlement britannique et la dévolution est proposée aux Gallois alors que le sentiment nationaliste, dans la seconde moitié de la décennie, s'essouffle au pays de Galles.

\section{Plaid Cymru, une véritable force politique à Londres}

4 Les années 1970 représentent une décennie de succès, tant dans les urnes que sur le plan politique : Plaid Cymru est alors une véritable force politique à Londres.

\section{Victoires électorales}

5 Après un premier mandat, Evans perd son siège lors des élections législatives en 1970, alors que le parti obtient trois fois plus de suffrages qu'en 1966, progressant de $4,3 \%$ à $11,5 \%$. Sa défaite est due à une combinaison de facteurs : la multiplication d'incidents et d'actions terroristes fomentés par Mudiad Amddiffyn Cymru (« Movement for the Defence of Wales ») et la Free Wales Army (FWA), l'action menée par Cymdeithas yr Iaith, association de défense de la langue galloise, et le sentiment, dans la circonscription de Carmarthen qu'Evans est davantage le député du pays de Galles que de la région. Après sa victoire en 1966, il est en effet surnommé "The Member for Wales ». Le mouvement républicain Mudiad Amddiffyn Cymru, surnommé MAC par ses partisans ${ }^{9}$, compte à son actif de nombreuses attaques contre des biens perpétrées entre 1963 et 1969. Quant à la FWA, elle fait une première apparition remarquée le 21 octobre 1965 lors de l'inauguration du barrage de Tryweryn ${ }^{10}$. C'est une petite armée plutôt organisée, dirigée par Julian Cayo Evans et visant essentiellement des bâtiments publics ainsi que des réservoirs ou des barrages. En février 1969, neuf personnes sont arrêtées lors d'une opération policière de grande envergure et mises en examen pour leurs activités terroristes au sein de la FWA en vertu du Public Order Act. Elles sont jugées en avril et la cour rend son verdict le $1^{\text {er }}$ juillet 1969, jour de l'investiture du Prince Charles : Julian Cayo Evans est condamné à 15 mois 
d'emprisonnement. Les années 1960 sont ainsi le théâtre d'une violence tout à fait nouvelle au pays de Galles ${ }^{11}$.

Evans est de retour au Parlement en octobre 1974 lorsque le parti emporte trois sièges, après en avoir obtenu deux en février. Fort de ses trois députés - Gwynfor Evans, Dafydd Wigley et Dafydd Elis Thomas - qui siègent dans une Chambre des Communes contrôlée, même si à une courte majorité, par les Travaillistes, Plaid Cymru se trouve dans une position idéale pour demander la mise en place d'une assemblée galloise à la suite de la publication du rapport Kilbrandon en 1973. Dès 1970, Evans est convaincu que la décennie qui s'ouvre sera synonyme de succès, et, confiant, il déclare le 2 janvier 1970 dans The Carmarthen Times: "The seventies decade will see Wales gain further freedom and gain her seat at the United Nations. ${ }^{12}$ " Les victoires électorales remportées par le parti nationaliste lui permettent de porter la voix galloise à Londres :

7 With Labour in power (with only a majority of 3), Plaid Cymru and the SNP were in an apparently ideal position. As he headed for Westminster, Gwynfor believed in all sincerity that an Assembly was imminent and that his life's work would be complete within three years ${ }^{13}$.

Plaid Cymru espère alors remporter des succès politiques.

\section{Succès politiques}

9 Aux yeux d'une partie de ses membres, le parti nationaliste gallois remporte déjà une première victoire le 17 mai 1967 lorsque la Reine Elizabeth II annonce que Charles, son fils aîné, qui porte le titre de Prince de Galles depuis juillet 1958, sera officiellement investi à Caernarfon le $1^{\text {er }}$ juillet 1969 , renouant ainsi avec une tradition ancienne. L'héritier de la couronne porte ce titre depuis 1301, lorsqu'Edouard II le reçut de son père Edouard I ${ }^{\mathrm{er}}$. Ce dernier voulait resserrer les liens entre l'Angleterre et le pays de Galles, région qu'il venait de conquérir. La cérémonie d'investiture du Prince de Galles se déroule au château de Caernarfon pour la première fois en 1911 pour le fils aîné du Roi Georges V, le futur Edouard VII. Après l'élection d'Evans en juillet 1966, et surtout la catastrophe d'Aberfan ${ }^{14}$ en octobre, le Welsh Office demande qu'une date soit rapidement fixée afin de renforcer l'union. L'annonce de la date de l'investiture soulève l'enthousiasme au pays de Galles, à l'instar de I.B. Griffith qui, dans la colonne qu'il rédige pour $Y$ Cymro, célèbre l'événement à venir : «A new member of the family is coming to Wales - is coming here to Caernarfon. We shall have a new son, and so let us show him and his mother and father what the family can do. ${ }^{15}$ " Les premières réactions des associations militantes à l'annonce de la date de la cérémonie sont cependant plutôt hostiles, ainsi celle de Dafydd Elis Thomas, alors président de la branche des jeunes au sein de Plaid Cymru : "If you don't want to make a fool of yourself in the greatest farce of Welsh history, don't come to Caernarfon in 1969 - but go back to Cambridge, Charlie boy. Wales has her own leaders and destiny now. ${ }^{16}$ » Toutefois, la décision même d'organiser, pour la seconde fois seulement au XXe siècle, une cérémonie au cœur du pays de Galles au moment où Plaid Cymru gagne du terrain montre le poids du nationalisme à la fin des années 1960. Buckingham va plus loin en décidant d'envoyer le jeune prince passer un semestre au University College of Wales à Aberystwyth, semestre au cours duquel il apprend le gallois. Le Prince Charles se trouve entouré de fervents nationalistes, désireux de faire en sorte qu'il s'identifie au pays de Galles : «It will be something to have a King of England who speaks Welsh. ${ }^{17}$ " Le parti autorise son vice-président, Tedi Millward, à jouer le rôle de tuteur à l'égard du prince. Il le sensibilise aux intérêts de la nation galloise et, dans un courrier confidentiel adressé à Evans, écrit sur son protégé : 
His views on the Welsh language are completely sound; he maintains that it should not be a political issue and that no one should make political capital out of it. He told me that he feels increasingly nationalistic the more he hears talk of internationalism and large units. Today, he received the three Black Books ${ }^{18}$ and the Voice of Wales ${ }^{19}$ and the water pamphlet ${ }^{20}$ - "I'd better hide them or people will think I've joined Plaid Cymru"21. least - with the awakening in Wales. I think it is perfectly possible that he will say something in favour of the language, for example, that can do great good.22 " Ce rapprochement du Prince Charles et des nationalistes gallois n'est pas sans inquiéter les instances londoniennes.

11 De plus, Plaid Cymru espère obtenir de Westminster la création d'une assemblée pour le pays de Galles. La commission Kilbrandon publie son rapport en 1973, après avoir consulté les principaux partis politiques, dont les nationalistes. Elle préconise quatre options, reflétant ainsi les profondes divergences d'opinion entre ses membres : la mise en place d'assemblées législatives en Écosse et au pays de Galles, d'une assemblée en Écosse mais seulement d'un conseil consultatif au pays de Galles, de conseils consultatifs dans les deux régions ou la mise en œuvre d'une dévolution exécutive dans toutes les régions britanniques. Lorsque le Parti travailliste revient au pouvoir en février 1974 avec seulement quatre sièges de plus que les Conservateurs, il a besoin du soutien des partis nationalistes et décide de s'engager sur la voie de la dévolution. Il publie dès septembre 1974 un premier livre blanc intitulé Democracy and Devolution. Proposals for Scotland and Wales, recommandant la mise en place d'assemblées en Écosse et au pays de Galles, toutes deux dépourvues de pouvoirs en matière fiscale, mais en précisant que si l'assemblée écossaise bénéficiera de pouvoirs législatifs, celle du pays de Galles n'aura que des pouvoirs exécutifs. En novembre 1975 sort un second livre blanc, Our Changing Democracy: Devolution to Scotland and Wales, qui inspire le projet de loi sur la dévolution introduit en décembre 1976, la Scotland and Wales Bill. L'objectif est de donner davantage de pouvoirs à la périphérie celtique tout en préservant le centre et l'union. Le gouvernement travailliste, dirigé jusqu'en mars 1976 par Harold Wilson, puis par James Callaghan, cherche à s'assurer du soutien des partis nationalistes: "Desperate to stay in office, Callaghan was keen to appease the MPs of the SNP and Plaid Cymru and to see the devolution proposals through with as few quibbles as possible. ${ }^{23}$ " Le projet de loi est néanmoins rejeté à la Chambre des Communes le 22 février 1977 par une majorité de 29 voix, alors que le gouvernement a concédé la tenue d'un référendum pour valider la dévolution. Il échappe le 23 mars à une motion de censure déposée par le Scottish National Party (SNP) et Plaid Cymru, furieux de l'échec du projet de loi, et signe un accord avec le Parti libéral. Fin mars, un second projet de loi est élaboré par Michael Foot, en charge de la dévolution, qui choisit cette fois-ci de différencier l'Écosse et le pays de Galles en proposant deux projets distincts. Le Wales Act est adopté en février 1978.

Gwynfor Evans, qui sympathise très vite avec Michael Foot, est enthousiaste et optimisme. Il considère que 1978 sera l'année du nationalisme gallois : « the most important year in Welsh history, at least for many centuries ${ }^{24} »$. Le référendum est organisé le $1^{\mathrm{er}}$ mars 1979. Evans est convaincu que la présence de trois députés nationalistes gallois à Westminster a permis d'obtenir cette victoire politique.

13 Enfin, la pression exercée par les nationalistes au Parlement s'illustre lors du Discours de la Reine prononcé le $1^{\mathrm{er}}$ novembre $1978^{25}$. Celle-ci mentionne de nombreux projets et décisions à venir pour le pays de Galles, notamment en faveur du bilinguisme, décrits par 
un journaliste du Financial Times ainsi : « unprecedented Welsh proposals ${ }^{26}$ ». Un journaliste du Western Mail, quotidien gallois, explique dans The Welsh Nation qu'il n'a jamais rien vu de tel au cours des 32 années passées à la Chambre des Communes ${ }^{27}$.

Les années 1970 sont par conséquent une décennie de succès pour le nationalisme gallois qui doit pourtant faire face à de nombreuses difficultés : des tensions et dissensions au sein de Plaid Cymru d'une part, l'essoufflement du sentiment nationalisme au pays de Galles d'autre part.

\section{De nombreuses tensions et dissensions au sein de Plaid Cymru}

15 Gwynfor Evans, considéré comme "The Member for Wales", se retrouve néanmoins isolé sur plusieurs sujets, sources de dissensions au sein de Plaid Cymru : la question de l'usage de la violence pour obtenir davantage d'autonomie pour le pays de Galles, l'entrée, puis le maintien, du Royaume-Uni dans le Marché commun et le projet de dévolution proposé après 1975 par le gouvernement travailliste.

\section{L'usage de la violence}

Les activités de MAC et de la FWA placent Plaid Cymru dans une position délicate, ce que souligne Philip Allan Butt, qui insiste sur les effets négatifs de ces comportements radicaux pour le parti nationaliste : « There was, however, very little the Blaid's advisers on publicity could do about the disastrous image that accrued to the party as a result of the series of bomb explosions which rocked Wales between 1966 and 1969..8 " Evans prônant depuis toujours le pacifisme, il ne peut cautionner l'usage de la violence, tout en étant lui-même impuissant face aux décisions prises par le gouvernement britannique, notamment l'inondation de plusieurs vallées pour approvisionner en eau l'Angleterre. Ainsi, au moment de la construction du réservoir de Tryweryn dans les années 1960, lieu du premier attentat au pays de Galles - perpétré dans le but de retarder les travaux - le parti ne peut empêcher le vote à Westminster de la loi autorisant la réalisation du site. Emrys Roberts, alors secrétaire général de Plaid, résume ce dilemme dans un article publié dans le Western Mail le 12 décembre 1963 :

Any widespread campaign of violent action in Wales today would be morally justifiable and politically foolish. It would alienate rather than win support. We in Plaid Cymru have nothing to do with it. [...] Violent action cannot be justified if it is a case of a minority trying to force its views on the majority of Wales. But when, as in the case of Tryweryn, it is undertaken in an attempt to force the government to respect the wishes of the people of Wales, it has ample justification ${ }^{29}$.

17 La position de Plaid Cymru devient encore plus difficile à tenir à partir de la fin des années 1960 lorsque le fragile équilibre instauré en Irlande du Nord se brise. Les antinationalistes estiment rapidement que l'existence d'une assemblée dévolue à Stormont en est la cause, et comparent nationalisme irlandais et gallois, ce que souligne Evans dans ses mémoires :

They [Anti-Nationalists] also tried to claim that the IRA demonstrated the perils of Welsh Nationalism. Time after time Wales was warned by George Thomas that campaigning for the Welsh language, though the methods used were completely non-violent, was sure to lead to a situation of terror like the one in Northern Ireland ${ }^{30}$. 
La situation reste semblable dans les années 1970, créant des tensions au sein du camp nationaliste entre partisans d'un militantisme plus radical, à l'image de Saunders Lewis, lui-même impliqué en 1936 dans l'incendie de l'école militaire à Penyberth, sur la péninsule de Llŷn, et défenseurs d'une action constitutionnelle, telle que préconisée par Evans $^{31}$. Ce dernier apporte donc après 1974 un soutien sans faille au gouvernement travailliste, seul moyen, selon lui, d'obtenir la mise en place d'une assemblée.

\section{Plaid Cymru et la CEE}

Lorsque le gouvernement conservateur dirigé par Edward Heath décide de candidater à nouveau pour l'entrée du Royaume-Uni au sein de la Communauté économique européenne (CEE), il publie le 7 juillet 1971 un livre blanc The United Kingdom and the European Communities, présentant point par point les avantages que représente la CEE, et se lance dans une campagne pour l'Europe. Heath rencontre en mai le Président français Georges Pompidou. Au fur et à mesure de l'avancée des négociations, Plaid Cymru doit définir sa position et le parti, Evans en tête, décide de s'opposer au projet européen, expliquant sa décision ainsi : " on the grounds that such a body would damage peace and the Welsh economy ${ }^{32} »$. Gwynn Matthews, membre de l'exécutif, rédige l'une des seules publications du parti sur le sujet, étudiant les répercussions économiques, financières et politiques de l'entrée du Royaume-Uni pour le pays de Galles. Il résume dans l'avantpropos le point de vue de Plaid Cymru:

This pamphlet is intended to explain the attitude of Plaid Cymru to the current membership negotiations between the British government and the member states of the European Economic Community. The policy of Plaid Cymru is to oppose Britain's entry into the European Community Market at this time because of adverse effect such an event would have on the material and cultural life of Wales ${ }^{33}$.

Matthews semble ainsi être le seul membre du parti à porter un intérêt au débat. Plaid Cymru se positionne contre la CEE en argumentant que le pays de Galles serait désavantagé en rejoignant une association que le parti considère comme antidémocratique et capitaliste ${ }^{34}$. En outre, Evans, pacifiste de la première heure, regarde la CEE comme une coalition militaire mettant en danger la stabilité internationale ${ }^{35}$. Plaid Cymru n'est pas opposé à l'idée d'une Europe unie, mais est plutôt favorable à une union sur le modèle de la European Free Trade Association (EFTA). Seulement une telle décision est prise sans véritable concertation au sein du parti. De ce fait, des membres importants de Plaid Cymru, nationalistes célèbres, s'expriment en faveur de la CEE, à l'instar de Saunders Lewis.

Début 1975, un référendum sur la CEE est imminent. La position du parti nationaliste est clairement illustrée par ce slogan: «Europe Yes, Common Market No». Il établit une distinction entre la CEE et l'Europe elle-même, ce qu'indique clairement Gwynfor Evans dans son ouvrage A National Future for Wales, publié en janvier 1975, soit quelques mois seulement avant le référendum. Il décrit la CEE en ces termes: " a centralising measure whose economic consequences for us would be to make Wales the periphery of a periphery ${ }^{36}$ ". Il justifie donc l'hostilité de Plaid Cymru par la centralisation et l'éloignement du pouvoir décisionnel, tout en reconnaissant l'importance de la dimension européenne puisqu'il ajoute: "Plaid Cymru has always been strongly European in outlook, seeing Wales as a part of European civilisation..$^{37} »$ Il décide alors de faire campagne pour le Non, ce qui est à nouveau source de tensions au sein du parti. Saunders Lewis accuse Plaid Cymru de soutenir le 
gouvernement de Londres: "for voting in favour of Westminster sovereignty and against Europe $^{38}$ ", stratégie selon lui paradoxale pour un parti nationaliste. Dafydd Wigley, député à Westminster, va à l'encontre de la ligne du parti en faisant ouvertement campagne pour le Oui, aux côtés des Conservateurs, de certains Travaillistes et des Libéraux, contrairement à Dafydd Elis Thomas, troisième député gallois, qui dirige la campagne nationaliste pour le Non, conjointement avec Neil Kinnock. De telles positions illustrent les désaccords de plus en plus flagrants entre les deux hommes. Le parti envoie ainsi un message très ambigu à ses électeurs, ce qui contribue à le discréditer. Le référendum a lieu le 5 juin 1975 et le pays de Galles se prononce à deux contre un en faveur du Oui, même dans les régions galloisantes, bastions traditionnels de Plaid cymru. Un article publié dans The Liverpool Daily Post le 7 juin montre à quel point le parti est ainsi désavoué par son électorat :

The Yes majorities in both Wales and Scotland were a severe rebuff for the Nationalist opponents of the Market. Both Plaid Cymru and the SNP saw the referendum as a fortuitous means of demonstrating that their countries wanted separatism. They too, have been given their verdicts ${ }^{39}$.

Face à ces résultats, Plaid opère un véritable virage stratégique et adopte une nouvelle politique: il réclame que le pays de Galles devienne membre du Commonwealth en obtenant le statut de dominion, au sein de l'Europe : « to campaign for full national status for Wales, within Europe $e^{40} »$. Il suit cette politique jusqu'en 2003.

\section{Un projet de dévolution tant attendu}

Après avoir réclamé pendant des années la création d'une assemblée et l'octroi de pouvoirs décisionnels, le parti nationaliste ne peut que se réjouir de la publication des deux livres blancs sur la dévolution, mentionnés précédemment. Evans, particulièrement enthousiaste, est considéré comme: "the man who would transform the white paper on devolution into an Assembly ${ }^{41}$ ». Il réserve un très bon accueil au second livre blanc sans même consulter le parti, alors que les membres de l'exécutif rejettent unanimement les propositions faites, qu'ils jugent décevantes. Lors d'une réunion tenue à Llandridod, un vote est organisé sur une motion d'Arfon pour rejeter les propositions du livre blanc ${ }^{42}$. La dévolution, pourtant au cœur des revendications de Plaid Cymru, est ainsi source de tensions et place le parti face à un nouveau dilemme : comment ne pas soutenir un projet de création d'une assemblée pour le pays de Galles, si minimaliste soit-il ? C'est la position défendue par Evans dans une lettre adressée à Dafydd Orwig Jones en février 1978 :

The people of Wales would not understand it at all if we voted against the bill. The government could easily blame us then for defeating it. Nothing would please them more. We all feel furious about the whole thing here but there isn't a lot we can do ${ }^{43}$

Ainsi, Evans, qui apporte son soutien au gouvernement travailliste, est de plus en plus isolé au sein de son propre parti, lui-même coupé de sa base électorale. En mai 1978, Dafydd Wigley lance un appel à l'unité :

The internal debate must end and we must begin to fight for Wales rather than fighting each other... It isn't enough to pronounce self-righteously about the referendum: "it has failed but our hands are clean because that's not what we wanted, in any case." Not only will Plaid lose but Wales will too ${ }^{44}$.

Malgré les succès que le parti remporte, il souffre de profondes divisions internes, et semble se couper de ses électeurs. 


\section{Le paradoxe du nationalisme gallois : Londres vs pays de Galles}

\section{La fracture entre Plaid Cymru et le pays de Galles}

Plaid Cymru est confronté à un véritable paradoxe : la dévolution est proposée aux Gallois alors que le nationalisme, dans la seconde moitié des années 1970, s'essouffle au pays de Galles ${ }^{45}$. À l'automne 1976, les journalistes utilisent même l'expression "The Great Devolution Bore " pour décrire le faible intérêt porté par les Gallois aux débats sur le projet de dévolution en discussion à Westminster. Des sondages réalisés à cette époque indiquent que seulement $27 \%$ des personnes interrogées sont favorables au premier projet de loi, et $31 \%$ des partisans de Plaid Cymru se déclarent indifférents ou tout simplement opposés ${ }^{46}$. Un sondage réalisé le 9 février 1979 par BBC Wales révèle que le pourcentage des sondés favorables à la mise en place d'une assemblée a chuté de 38 à $33 \%$ depuis septembre 1978. Les Conservateurs, pourtant à l'origine de la création du poste de Ministre aux affaires galloises, puis de Secretary of State for Wales, s'opposent au projet et suivent en cela l'opinion publique. Une étude menée par le Parti conservateur montre en effet que la population n'est absolument pas intéressée par la dévolution : «It was really an in-group subject - the Press, TV and politicians were interested, people were not. ${ }^{47} »$ En janvier 1975, Nicholas Edwards, député pour le Pembrokeshire ${ }^{48}$, adresse un courrier à Margaret Thatcher, sur le point de prendre la tête du Parti conservateur, dans lequel il évoque même l'hostilité évidente des Gallois au projet ${ }^{49}$. Un sondage réalisé conjointement par le Western Mail et HTV une semaine avant le référendum du $1^{\mathrm{er}}$ mars 1979 indique que seulement $22 \%$ des personnes interrogées sont favorables à la dévolution. Les opposants justifient leur position par différents arguments : $61 \%$ estiment que l'assemblée sera trop coûteuse, pour $43 \%$ elle ne sera qu'une nouvelle strate de bureaucratie, et, pour $40 \%$, elle sera utilisée par les nationalistes pour rompre avec le Royaume-Uni ${ }^{50}$. Sur les 23 députés travaillistes que compte alors le pays de Galles, 12 se déclarent en faveur d'une assemblée, mais seulement six d'entre eux décident de soutenir activement la Wales for the Assembly Campaign (WAC), craignant d'être associés aux nationalistes. Six députés travaillistes choisissent même de faire campagne pour le Non. Le «Gang des Six », mené par Neil Kinnock et Leo Abse, deux députés du Gwent, prônent l'unité de la classe ouvrière, considérant le centralisme comme condition à la mise en place du socialisme, et brandissent le spectre d'une domination de la future assemblée par les galloisants ${ }^{51}$. Evans, ne comprenant pas qu'un député gallois puisse s'opposer à la création d'une assemblée pour le pays de Galles, dénonce avec virulence l'attitude de Neil Kinnock, qui devait devenir en octobre 1983 président du Parti travailliste. Denis Balsom considère que la campagne ne reçoit le soutien que des Gallois sensibles aux arguments de Plaid Cymru, justifiant ainsi le faible taux de participation au référendum :

Thus having identified the interests of the nationalists with devolution, the Welsh electorate would appear to have accorded the issue the same derisory level of support as that which the nationalists manage to secure at most elections ${ }^{52}$.

De ce fait, les députés nationalistes semblent déconnectés de la réalité du terrain et incapables de saisir l'opinion publique qui choisit de rejeter massivement $-79,7 \%$ des votants - le projet de dévolution proposé lors du référendum. C'est un véritable camouflet pour le parti nationaliste, et surtout pour son président, qui n'a cessé de 
soutenir le projet envers et contre tout. Plaid Cymru est alors coupé de sa base électorale. S'il est, dans la seconde moitié de la décennie, plus présent que jamais à Westminster, il s'est éloigné des préoccupations des Gallois :

Nationalism was certainly a force at Westminster, but this was almost entirely due to the problems faced first by Wilson and now, by Callaghan. On the contrary, in Wales, nationalism had become increasingly unpopular during early 1976, and Gwynfor and those around him virtually disregarded the fact that Kinnock, Abse and the anti-devolutionists had won over Welsh population opinion. They may have been demagogues, but the reality was that they understood Wales better than Plaid Cymru did ${ }^{53}$.

L'essoufflement du sentiment nationaliste dans les années 1970 s'explique tout d'abord par la multiplication des messages discordants envoyés par Plaid cymru, comme étudié précédemment, ensuite par le contexte économique difficile. Les Gallois, tout comme le reste de la population britannique, ont d'autres sujets de préoccupation tels que la crise économique, l'inflation, le chômage, les grèves, ce que souligne Charles Quant, journaliste au Liverpool Daily Post le 22 janvier 1979: «Do Mr and Mrs Jones of Wales really rate the Welsh (for or against the) Assembly as an issue to bear comparison with strikes, pickets and pay ${ }^{54}$ " Enfin, contrairement à l'Écosse, le pays de Galles ne compte que peu d'institutions civiques qui lui sont propres, et elles sont souvent plutôt récentes, à l'instar du Welsh office. Il est donc nécessaire de différencier la position de Plaid Cymru à Westminster et l'attrait du nationalisme au pays de Galles.

\section{La fragmentation du mouvement nationaliste gallois}

Plaid Cymru n'est pas le seul vecteur d'expression du sentiment nationaliste au pays de Galles. Alors que le parti, fondé à l'origine comme un groupe de pression défendant la langue et la culture galloises, devient une véritable force politique désireuse d'utiliser la voie constitutionnelle pour obtenir des avancées pour le pays de Galles, différentes associations se sont créées pour combler le vide laissé par le repositionnement de Plaid. Cymdeithas yr Iaith (Welsh Language Society), est ainsi mise en place à la suite de l'allocution radiophonique de Saunders Lewis le 13 février 1962 intitulée «Tynged yr Iaith» («The Fate of the language ») dans laquelle il appelle à la lutte et réclame un statut officiel pour le gallois, l'un des principaux objectifs de Plaid Cymru au moment de sa création. Ce mouvement, constitué en majorité d'étudiants - dont Meinir Evans, la fille de Gwynfor Evans, plusieurs fois emprisonnée pour ses activités au sein de l'association - prône la désobéissance civile, et encourage des actions directes contre des symboles de la domination anglaise. Ainsi, au cours de l'été 1978, Plaid Cymru et Cymdeithas yr Iaith se retrouvent en concurrence lors des débats sur le projet de loi pour la dévolution. Cymdeithas appelle à des actions militantes et son président, Rhodri Williams, est décrit en ces termes: «the most charismatic symbol of Welsh nationalism ${ }^{55}$ ». Ce n'est donc plus Evans, pourtant fêté une décennie plus tôt, qui possède un tel titre. L'association de défense de la langue refuse de faire campagne pour la dévolution, expliquant que son implication nuirait à son indépendance et constituerait une perte de temps vu le projet proposée ${ }^{56}$. Après l'échec des référendums écossais et gallois, le SNP annonce son intention de déposer une motion contre le gouvernement de Callaghan. Evans, fidèle à ses convictions, décide de ne pas aider les Conservateurs à faire tomber le gouvernement, ce qui n'empêchera pas sa chute le 22 mars 1979, à une voix. 


\section{Conclusion} remporte, est devenu une véritable force politique à Westminster, le parti est néanmoins victime de tensions et dissensions internes. Les membres ne cachent pas leurs désaccords sur des sujets comme l'usage de la violence pour faire avancer la cause galloise, l'entrée, puis le maintien, du Royaume-Uni au sein de la CEE et, thème central, le projet de dévolution proposé par le gouvernement travailliste. De telles divisions contribuent à brouiller le message envoyé à l'électorat et à décrédibiliser le parti, en rupture avec sa base. Enfin, elles conduisent à une fragmentation du mouvement nationaliste gallois, différentes associations se positionnant en concurrence avec le parti, lui-même divisé. En témoignent ces lignes de Saunders Lewis dans une lettre adressée juste avant les résultats des élections qui suivent la chute du gouvernement Callaghan à Y Faner et publiée le 4 mai 1979 : «I hope that these three [MPs] will have their due on the third of May, but Wales is more important to me than Plaid Cymru, and it is not through that party, with its present leadership, and its present policy, that Wales will have self-government..$^{57}$ "

Après l'échec du référendum du $1^{\mathrm{er}}$ mars 1979 et les mauvais résultats obtenus aux élections, le parti est moribond et doit se reconstruire, dans un contexte politique dominé par les Conservateurs: "Devolution was dead in the water, and with Thatcher as Prime Minister, nationalists feared it was only a matter of time before the Welsh language went the same way. Gwynfor was cut to the quick; rejected, he entered his own dark night of the soul ${ }^{58} »$. Plaid Cymru choisit en 1981 un nouveau président, et doit attendre 18 ans, et l'arrivée de Tony Blair au pouvoir en 1997, pour qu'un projet de dévolution soit présenté. Aujourd'hui, le parti est de nouveau sur le devant de la scène à l'Assemblée, concluant en mai 2016 un accord avec les Travaillistes gallois dirigé par Carwyn Jones pour permettre à celui-ci de conserver son poste de First Minister, et un élargissement de la dévolution est proposé aux Gallois alors que le parti nationaliste n'est arrivé qu'en quatrième position lors des élections législatives de mai 2015..

Stéphanie Bory est Maître de Conférences en civilisation britannique à l'Université Jean Moulin Lyon 3 depuis 2009 et membre de l'Institut d'Etudes Transtextuelles et Transculturelles (IETT). Elle travaille sur l'écologisme et le nationalisme, notamment au pays de Galles. Après avoir soutenu une thèse sur la politique environnementale de l'Assemblée galloise, elle a publié différents articles sur ces sujets et participé à de nombreux colloques. Elle s'intéresse aussi aux institutions galloises, en particulier depuis la dévolution.

\section{BIBLIOGRAPHIE}

Anon, « 1 a.m.: The Welsh Unseat Labour », The Daily Mail, 15-07-1966. 
Butt Philip Allan, The Welsh Question. Nationalism in Welsh Politics. 1945-1970, Cardiff, University of Wales Press, 1975.

Conservative Party, Minutes of the Conservative Study Group on Devolution, 16-01-1975, Lord Crickhowell Papers, National Library of Wales.

Cymdeithas yr Iaith, Minutes of Cymdeithas yr Iaith Parliament, 13-01-1979, Cymdeithas yr Iaith Gymraeg Papers, Boîte 11, New Library of Wales.

Elis Thomas Dafydd, The Sunday Express, 07-01-1968.

Evans Gwynfor, Save Cwm Tryweryn for Wales, Cardiff, Plaid Cymru, 1956.

Evans Gwynfor, A National Future for Wales, Cardiff, Plaid Cymru, John Penry Press, 1975.

Evans Gwynfor, For the sake of Wales. The Memoirs of Gwynfor Evans, traduit par Meic Stephens, Cardiff, Welsh Academic Press, 1996.

Evans Rhys, Gwynfor Evans, Portrait of a Patriot, Talybont, Y Lolfa, 2008.

Foulkes David, Barry Jones J. \& Wilford R.A., The Welsh Veto. The Welsh Act 1978 and the Referendum, Cardiff, University of Wales Press, 1983.

Great Britain, The United Kingdom and the European Communities, Londres, HMSO, Cmnd 4715, 1971.

Great Britain, Report and Memoranda of the Royal Commission on the Constitution, Londres, HMSO, Cmnd 5460, 1973-1974.

Great Britain, Democracy and Devolution. Proposals for Scotland and Wales, Londres, HMSO, Cmnd 25608, septembre 1974 .

Great Britain, Our Changing Democracy: Devolution to Scotland and Wales, Londres, HMSO, Cmnd 6348, novembre 1975.

Griffith I.B., Y Cymro, 01-06-1967.

Gwenallt, « Sir Gaerfyrddin », Y Draig Goch, septembre 1967.

Lewis Saunders, Y Faner, 04-05-1979.

Matthews Gwynn, Wales and the European Common Market, Cardiff, Plaid Cymru, 1970.

Quant Charles, Liverpool Daily Post, 22-01-1979.

Queen's Speech, HC Deb, 01-11-1978, vol. 957, cc4-8, http://www.hansard.millbanksystems.com/ commons/1978/nov/01/queens-speech, consulté en juillet 2016.

Sked Alan \& Cook Chris, Post-War Britain, A Political History 1945-1992, Londres, Penguin, 1993 (4 edition, $1^{\mathrm{e}}$ edition en 1979).

Thomas Wyn, Hands Off Wales, Nationhood and Militancy, Llandysul, Gomer Press, 2013.

Turner Alwyn W., Crisis? What Crisis? Britain in the 1970s, Londres, Aurum, 2013.

\section{NOTES}

1. Titre inspiré de la déclaration de Rhys Evans, Portrait of a Patriot, Talybont, Y Lolfa, 2008, p. 340.

2. Les eisteddfods sont des concours de musique et de poésie organisés chaque année au pays de Galles, de tradition ancienne, mais relancés au début du XIX ${ }^{\mathrm{e}}$ siècle par Iolo Morganwg.

3. « [a] Welsh-speaking Wales », expression fréquemment utilisée par Saunders Lewis. 
4. Cymru Fydd est une société patriotique fondée en 1886 par quelques Libéraux, dont Thomas Edwards Ellis et David Lloyd George, futur Premier ministre britannique. Elle demande l'autonomie pour le pays de Galles.

5. « The Nationalist Dawn ».

6. Voir Rhys Evans, Gwynfor Evans, Portrait of a Patriot, p. 268.

7. «The Royal Commission on the Constitution ».

8. Report and Memoranda of the Royal Commission on the Constitution, Londres, HMSO, Cmnd 5460, 1973-1974.

9. Ce sigle reprend le nom gallois et leur permet de se moquer de Harold Macmillan, Premier ministre de janvier 1957 à octobre 1963, également appelé «Super-Mac ». Ce surnom est repris par Alan Sked et Chris Cook dans Post-War Britain, A Political History 1945-1992, Londres, Penguin, $4^{\mathrm{e}}$ édition, 1993, p. 160.

10. La construction de ce barrage, inauguré en 1965, nécessite le déplacement des 70 habitants du village de Capel Celyn, alors englouti sous les eaux.

11. Voir Wyn Thomas, Hands Off Wales, Nationhood and Militancy, Llandysul, Gomer Press, 2013. L'auteur étudie les campagnes militantes menées au pays de Galles dans les années 1960, plus particulièrement celles de MAC et de la FWA, à travers de nombreux entretiens.

12. Cité dans Rhys Evans, op. cit., p. 308.

13. Rhys Evans, op. cit., p. 340.

14. Le 21 octobre 1966, à 9h15 le matin, un terril du Merthyr Vale Colliery s'effondre, et le glissement de terrain provoque une avalanche noire qui recouvre le village, notamment l'école, faisant près de 150 victimes, dont 116 enfants. Environ 70 catastrophes se produisent dans les mines galloises entre 1837 et 1934, mais aucune d'entre elles ne marque autant les esprits que celle d'Aberfan. Le rapport publié en août 1967 de la commission parlementaire chargée d'enquêter sur les causes de l'accident établit clairement la responsabilité du National Coal Board puisque le terril a été construit sur un ruisseau.

15. I.B. Griffith, Y Cymro, 01-06-1967.

16. Dafydd Elis Thomas, The Sunday Express, 07-01-1968.

17. Propos tenus par Gwynfor Evans devant le comité exécutif de Plaid Cymru le 4 novembre 1967, Ioan Bowen Rees Papers, National Library of Wales.

18. C'est ainsi que sont qualifiés les ouvrages compilant toutes les questions posées à l'écrit et à l'oral par Evans à Westminster: "In imitation of the Government's habit of publishing its policies in White Papers, I too published a selection of my questions, together with the damning answers given by the Government, under the title Black Papers on Wales, in which the Government stood condemned out of its own mouth ", Gwynfor Evans, For the sake of Wales. The Memoirs of Gwynfor Evans, traduit par Meic Stephens, Cardiff, Welsh Academic Press, 1996, p. 185.

19. Titre de l'ouvrage rassemblant les discours prononcés par Gwynfor Evans à la Chambre des Communes. Plusieurs volumes seront publiés.

20. Il s'agit de Save Cwm Tryweryn for Wales, publié par Gwynfor Evans en 1956, afin de dénoncer le projet de construction du barrage de Tryweryn.

21. Lettre de Tedi Millward à Gwynfor Evans, 30-05-1969, Islwyn Ffowc Elis papers, New Library of Wales.

22. Loc. cit.

23. Alwyn W. Turner, Crisis? What Crisis? Britain in the 1970s, Londres, Aurum, 2013, p. 229.

24. Lettre de Gwynfor Evans à Rhys Davies, 05-01-1978.

25. En voici quelques extraits : "Legislation will be introduced to provide additional finance for the National Enterprise Board and for the Scottish and Welsh Development Agencies » (paragraphe 6), «Draft Orders will be laid early in the Session to provide for the referenda on devolution to the Scottish and Welsh Assemblies, to be held when the new electoral registers are available " (paragraphe 6), "Legislation will be introduced to improve the law on education in England and Wales and to enable grants to be made in 
Wales towards the cost of bilingual education » (paragraphe 7), Queen's Speech, HC Deb, 01-11-1978, vol. 957, cc4-8, http://www.hansard.millbanksystems.com/commons/1978/nov/01/queensspeech, consulté en juillet 2016.

26. Financial Times, 02-11-1978.

27. The Welsh Nation, décembre 1978.

28. Philip Allan Butt, The Welsh Question. Nationalism in Welsh Politics. 1945-1970, Cardiff, University of Wales Press, 1975, p. 188. Il ajoute, à propos des événements de Tryweryn : "The Tryweryn affair had placed Plaid Cymru in a quandary. The failure of the party's constitutional strategy was constantly exposed by the steady progress in building the reservoir in the Tryweryn valley. Welsh opinion and Welsh MPs had been disregarded, yet Plaid Cymru had been unable and unwilling to capitalize on this situation. In the period 1960-5 Plaid Cymru had to make the choice while the reservoir was being built of sponsoring direct action at the site (and risking great unpopularity) or of doing nothing (and losing face with its own supporters) », op. cit., p. 93.

29. Emrys Roberts, Western Mail, 12-12-1963.

30. Gwynfor Evans, op. cit., p. 183.

31. Dans ses mémoires, Evans explique son engagement politique ainsi : "I was driven into politics for the reason that it's in the political field that important decisions are made which affect the life of the Welsh nation ", op. cit., p. 181.

32. Rhys Evans, op. cit., p. 322. Lors du débat à la Chambre des Communes précédant la première candidature du Royaume-Uni, Evans s'exprime contre cette candidature, ce qu'il explique dans ses mémoires : "I argued against, basing my speech on two main points. One was the economic harm that British membership would cause to Wales and the other was the danger that the Community would develop into a great military power possessing nuclear weapons. I argued that such an eventuality might lead to a war that would destroy the civilization of Europe ", Evans, op. cit., p. 187.

33. Gwynn Matthews, Wales and the European Common Market, Cardiff, Plaid Cymru, 1970, avantpropos.

34. Rhys Evans, op. cit., p. 346.

35. Gwynfor Evans, Western Mail, 02-06-1975.

36. Gwynfor Evans, A National Future for Wales, Cardiff, Plaid Cymru, John Penry Press, 1975, p. 100.

37. Loc. cit.

38. Saunders Lewis, Western Mail, 21-01-1975.

39. Liverpool Daily Post, 07-06-1975.

40. Rhys Evans, op. cit., p. 348.

41. Ibid., p. 341.

42. The Liverpool Daily Post, 05-01-1976.

43. Lettre de Gwynfor Evans à Dafydd Orwig Jones, 20-02-1978, Gwynfor Evans Papers, 1979, Box 1, New Library of Wales.

44. Mémorandum de Dafydd Wigley, 18-05-1978, Plaid Cymru Papers L26, Plaid Cymru Papers.

45. "The irony, of course, is that devolution would be offered to the people of Wales at a time when nationalism was in decline in Wales and beyond », Rhys Evans, op. cit., p. 345.

46. Ibid., p. 358.

47. Minutes of the Conservative Study Group on Devolution, 16-01-1975, Lord Crickhowell Papers, National Library of Wales.

48. Il devient en février 1975, après l'élection de Margaret Thatcher à la tête du parti, Secretary of State for Wales fantôme jusqu'en 1979, puis Secretary of State for Wales sous les deux premiers gouvernements Thatcher. Il se retire en 1987 et entre à la Chambre des Lords avec le titre Lord Crickhowell.

49. Lettre de Nicholas Edwards à Margaret Thatcher, 19-01-1975, Lord Crickhowell Papers, National Library of Wales.

50. Loc. cit. 
51. Dans le Newport Argus du 27 février 1979, Leo Abse touche une corde sensible chez une population à 80\% monoglote: "Are the English speaking Welshmen and Welshwomen of Newport, Pontypool and Gwent to become second-class citizens in their own land? Is Newport to be governed from Cardiff by a huge Welsh speaking civil service?».

52. Denis Balsom, «Public Opinion and Welsh Devolution », in David Foulkes, J. Barry Jones \& R.A. Wilford, The Welsh Veto. The Welsh Act 1978 and the Referendum, Cardiff, University of Wales Press, 1983, p. 213.

53. Rhys Evans, op. cit., p. 357.

54. Charles Quant, Liverpool Daily Post, 22-01-1979.

55. Rhys Evans, op. cit., p. 373.

56. Minutes of Cymdeithas yr Iaith Parliament, 13-01-1979, Cymdeithas yr Iaith Gymraeg Papers, Boîte 11, New Library of Wales.

57. Saunders Lewis, Y Faner, 04-05-1979.

58. Rhys Evans, op. cit., p. 391.

\section{RÉSUMÉS}

Plaid Cymru, le parti nationaliste gallois créé en 1925, remporte le 14 juillet 1966 sa première victoire avec l'élection dans la circonscription de Carmarthen de Gwynfor Evans, dirigeant du parti depuis 1945, et devient ainsi une véritable force politique. Après les élections législatives d'octobre 1974, fort de trois députés (Gwynfor Evans, Dafydd Wigley et Dafydd Elis-Thomas) qui siègent au Parlement contrôlé (même si à une courte majorité) par les Travaillistes, le parti gallois est dans une position idéale pour obtenir une assemblée à la suite des recommandations du rapport Kilbrandon publié en 1973.

Pourtant, Plaid Cymru fait face à un paradoxe : la dévolution est proposée aux Gallois alors que le nationalisme, dans la deuxième moitié de la décennie, est en déclin, et que le parti lui-même est divisé sur plusieurs sujets. Après l'échec du référendum du $1^{\mathrm{er}}$ mars 1979 et les mauvais résultats obtenus aux élections législatives qui suivent la chute du gouvernement Callaghan, le parti est moribond et doit se reconstruire.

Plaid Cymru, the Welsh nationalist party set up in 1925, won its first seat on 14 July 1966 when Gwynfor Evans, the party leader since 1945, was elected MP for Carmarthen. It hence became a party to be reckoned with. After the October 1974 general election, the Welsh party, represented in the British Parliament by three MPs (Gwynfor Evans, Dafydd Wigley et Dafydd Elis-Thomas), was ideally placed to call for the creation of a Welsh assembly, especially after the publication of the Kilbrandon Report in 1973. And yet Plaid Cymru found itself in a paradoxical position: Welsh people were offered devolution at a time when nationalism, in the second half of the 1970s, was declining, and the party itself was divided on several issues. After the No vote in the March 1979 referendum on devolution and the poor results obtained by Plaid Cymru in the general election that followed the fall of the Callaghan government, the party had to rebuild itself. 
INDEX

Mots-clés : Pays de Galles, Plaid Cymru, dévolution, nationalisme

Keywords : Wales, Plaid Cymru, devolution, nationalism

\section{AUTEUR}

\section{STÉPHANIE BORY}

Institut d'Etudes Transtextuelles et Transculturelles (IETT), EA 4186, Université Jean Moulin Lyon 3 